\title{
Flächendeckende Entsorgung bis 2005. Liegt Brandenburg in der Zeit?
}

\author{
Lothar Ebner, Michael König, Andrea Wardacki, Sandra Giern
}

\section{Einführung}

Zu Beginn der Betrachtungen ist eine Auseinandersetzung mit dem Begriff „Abwasser“ sehr nützlich. Ist dieser Begriff überhaupt „richtig“? Die Antwort, die wir uns gaben, lautete: Ja und nein!

1. Ja - denn es ist ein Abfall, dessen wir uns entledigen wollen. Ob wir es objektiv müssen, sollten wir noch einmal überdenken.

2. Nein - da es ein Teil der uns umgebenden Natur ist, den wir uns zum Gebrauch entleihen, den wir aber aufbereitet der Natur wieder zufuihren müssen, um im regionalen Kreislauf nicht ein Defizit zu erzeugen. Über Nachhaltigkeit reden wir alle, aber handeln wir auch danach?

Der Begriff „Abwasserentsorgung“ entbindet uns nicht von der Verpflichtung des Kreislaufwirtschafts- und Abfallgesetzes, erst zu entsorgen, wenn wir keine umweltpolitische und wirtschaftliche Möglichkeit zur Verwertung mehr sehen. Schließen wir nicht mit vielen Formulierungen in Gesetzen und Verordnungen über die „Abwasserentsorgung“ diese Pflicht, nach Verwertungsmöglichkeiten zu suchen, von vornherein aus?

\section{2 Überblick}

Wir wollen in diesem Beitrag zu folgenden Problemen Stellung nehmen:

- gesetzlicher Hintergrund

- Anschlussgrad und Probleme bei der Erschließung

- Abwasserentsorgung und Abwasseranlagen in Brandenburg

- dezentrale Lösungen

- Miniclar - eine moderne Kleinkläranlage

- Zusammenfassung

- Ausblick

\section{Gesetzlicher Hintergrund}

Ausgehend von der Europäischen Richtlinie 91/271/EWG über die Behandlung von Kommunalem Abwasser [1] erfolgte zum geforderten Termin 30.06.1993 in der Bundesrepublik Deutschland keine formelle Umsetzung in nationales Recht, sondern es wurden Abwassergrenzwerte in die Einleiterbescheide übernommen und die erforderlichen Messprogramme durchgefuihrt.

Die durch die obige EG-Richtlinie geforderte Ausweisung empfindlicher Gebiete zum 31.12.1993 erforderte etli- che Mühen und wurde sogar von Brüssel angemahnt. Im weiteren Zeitplan standen bis 31.12.1998 die kanaltechnische Erschließung aller Verdichtungsgebiete mit mehr als 10.000 EWG und Abwassereinleitung in empfindliche Gebiete sowie die weitergehende Abwasserbehandlung für kommunale Abwässer. Bis zum 31.12. 2000 waren die kanaltechnische Erschließung aller Verdichtungsräume mit mehr als 15.000 EWG und sowohl die mechanische und als auch eine biologische Reinigung von Abwässern gefordert. Dieser Entwicklungsprozess soll bis zum 31.12.2005 für alle Gemeinden mit mehr als 2.000 EWG abgeschlossen werden.

\section{Entwicklung der Abwasserentsorgung in Brandenburg}

Um den Forderungen der Europäischen Richtlinie Rechnung zu tragen, wurden von der Landesregierung Brandenburg in der Kommunalabwasserverordnung vom 18.02.1998 [2] weitgehende Festlegungen getroffen:

- bis 31.12.1998 Errichtung von Kläranlagen mit weitergehender Abwasserbehandlung und ggf. Kanalisation in gemeindlichen Gebieten mit mehr als 10.000 EWG

- bis 31.12.2000 Errichtung von Kläranlagen für Abwassereinleitungen aus bestimmten Industriebranchen mit mehr als 4.000 EWG

- bis 31.12.2005 Kläranlagen mit biologischer Reinigung und ggf. Kanalisation in gemeindlichen Gebieten mit mehr als 2.000 EWG geeignete Abwasserbehandlung für kommunales Abwasser aus gemeindlichen Gebieten mit weniger als $2.000 \mathrm{EWG}$

Damit wurde ein ehrgeiziges Ziel gesetzt. Es ist nun an uns zu sehen, wie die Umsetzung erfolgt ist. Bis 2001 wurden mehr als $75 \%$ aller Gemeinden an kommunale Abwassernetze angeschlossen, wie wir der nachfolgenden Darstellung entnehmen können.

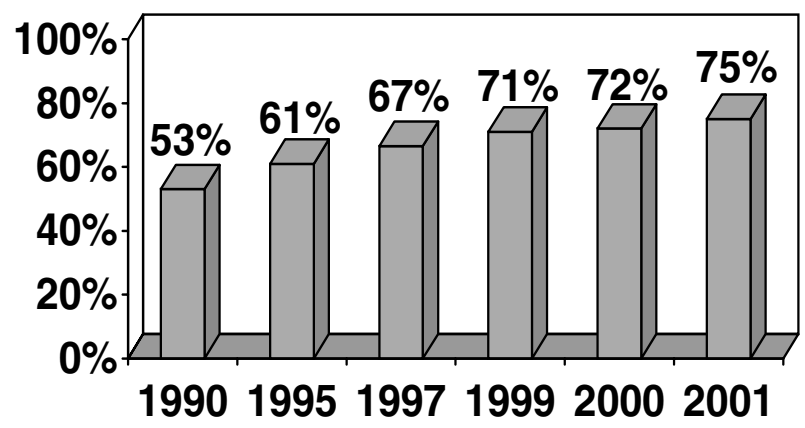

Abb. 1: Entwicklung des Anschlussgrades in Brandenburg nach [3] 
Natürlich gibt es bei der Erschließung vieler Gemeinden in diesem Prozess erhebliche Probleme, von denen wir hier einige nennen möchten:

- ca. $30 \%$ (800.000) der Brandenburger leben in Orten mit weniger als $2.000 \mathrm{EW}$,

- die oft ungenügenden Abwasseranlagen haben teilweise erheblichen Sanierungsbedarf,

- der Bau größerer Abwassernetze ist finanziell und technisch aufwendig,

- es müssen dezentrale Lösungen entwickelt und eingesetzt werden.

In diesem Zusammenhang stellt sich die Frage, welche Arten der Abwassernutzung in Brandenburg zur Zeit bestehen. Der nachfolgenden Grafik kann entnommen werden, dass zwei Drittel der Haushalte in Brandenburg an mechanisch-biologischen Anlagen mit Nährstoffreduzierung angeschlossen sind. Ca. 29 \% der Einwohner lassen ihr Abwasser zum jetzigen Zeitraum immer noch abfahren.

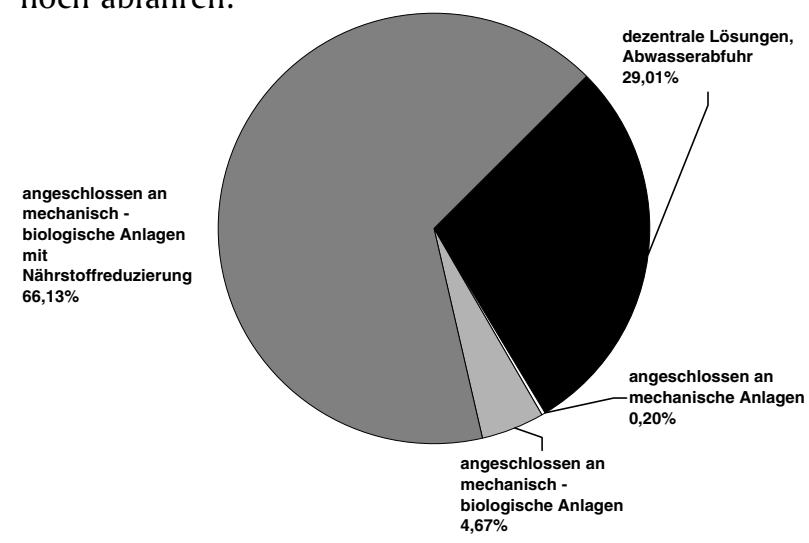

Abb. 2: In Brandenburg genutzte Arten der Abwasserentsorgung nach [3]

Durch den Neu- bzw. Umbau von Abwasseranlagen wurden allein in den Jahren 2000 und 2001 insgesamt 530 Anlagen mit Investitionssummen von über 508 Mio. DM, das sind 260 Mio. EUR, eingesetzt.

\begin{tabular}{|l|l|l|l|l|}
\hline \multirow{2}{*}{} & \multicolumn{2}{|c|}{2000} & \multicolumn{2}{c|}{2001} \\
\cline { 2 - 5 } & $\begin{array}{l}\text { genehmigte } \\
\text { Anträge }\end{array}$ & $\begin{array}{l}\text { Investitions- } \\
\text { umfang }\end{array}$ & $\begin{array}{l}\text { genehmigte } \\
\text { Anträge }\end{array}$ & $\begin{array}{l}\text { Investitions- } \\
\text { umfang }\end{array}$ \\
\hline $\begin{array}{l}\text { Kläranlagen, } \\
\text { Sanierung und } \\
\text { Umbau }\end{array}$ & 10 & 22,80 Mio. DM & 7 & 24,9 Mio. DM \\
\hline $\begin{array}{l}\text { Kläranlagen, } \\
\text { Neubau }\end{array}$ & 7 & 6,7 Mio. DM & 3 & 4,8 Mio. DM \\
\hline $\begin{array}{l}\text { Abwasserablei- } \\
\text { tungsanlagen }\end{array}$ & 272 & 188,5 Mio. DM & 231 & 260,3 Mio. DM \\
\hline gesamt & 289 & 218 Mio. DM & 241 & 290 Mio. DM \\
\hline
\end{tabular}

Neu- und Umbau von Abwasseranlagen - Förderung durch das MLUR nach [3]

Aus den oben genannten Tatsachen heraus (geringe Bevölkerungsdichte, Zustand der bestehenden Abwasseranlagen, geringe finanzielle Mittel) ergeben sich Vorteile beim Einsatz dezentraler Lösungen für die Abwasserentsorgung:

- es entfallen die Kosten für lange Rohrnetze,

- die Kapazität der Anlage kann besser genutzt werden, da sie der Zahl der angeschlossenen Personen angepasst wird,
- keine Mischung mit industriellen Abwässern.

Eine zeitgemäße und finanzgünstige Lösungsvariante von dezentralen Abwasseranlagen besteht in der Nutzung von Kleinkläranlagen. 1995 wurde vom Brandenburger MLUR das Förderprogramm „Grundstückskleinkläranlagen“ eingerichtet. Seitdem wurde der Bau von KKA für mehr als 20.000 Einwohner gefördert. 2000 wurden 4,5 Mio. DM für 769 Anträge bewilligt und ca. 3.000 Einwohner angeschlossen, 2001 wurden 4,9 Mio. DM für 844 Anträge bewilligt und ca. 3.500 Einwohner angeschlossen. Hier wurden also in den letzten beiden Jahren über 4,8 Mio. bewilligt und damit über 6.500 Einwohner Brandenburgs an eine kostengünstige Variante einer Abwasseranlage angeschlossen.

\section{Kleinkläranlage MINICLAR}

Ein Beispiel für diese Variante einer Kleinkläranlage ist die Kleinkläranlage MINICLAR [4], die als Beispiel für andere ähnliche Anlagen dient und hier kurz mit ihren Parametern vorgestellt werden soll:

- Bioreaktor mit aerober Nitrifikations- und anaerober Denitrifikationsphase,

- besonders hoher Wirkungsgrad (> $95 \%$ ),

- kann in unmittelbarer Nähe zum Gebäude platzsparend eingebaut werden,

- energiesparend durch Verwendung eines Generators und einer Mammutpumpe,

- liefert Brauchwasser und spart somit Trinkwasser.
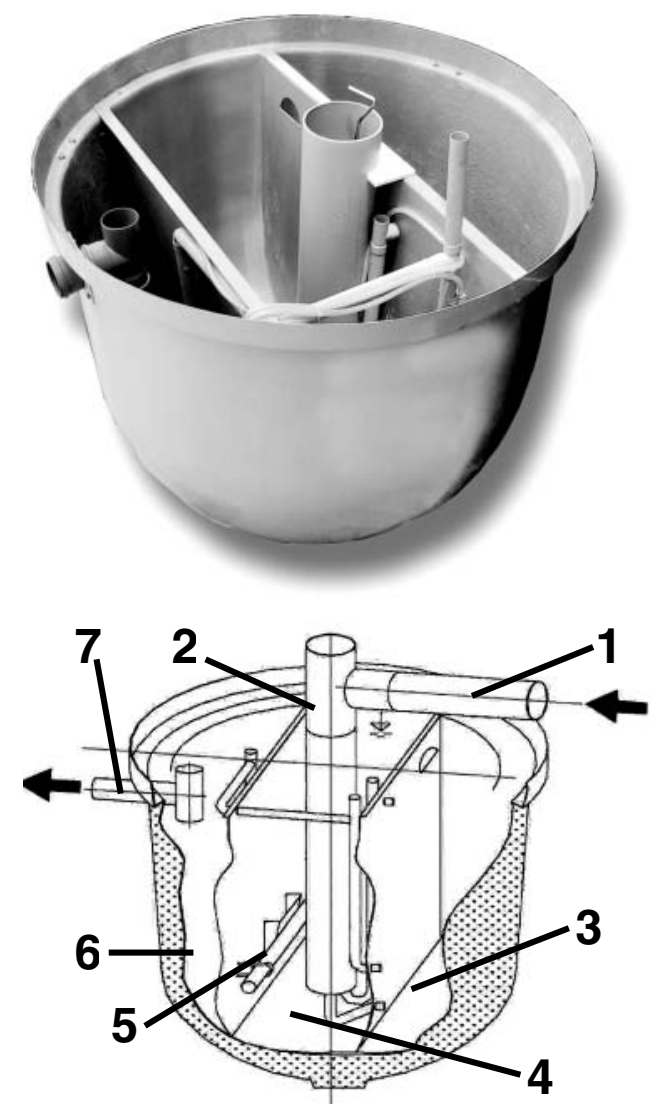

Abb. 3: Kleinkläranlage MINICLAR - Aufbau. (1) Zulauf, (2) Abwassereinlauf mit Rechenkorb, (3) 1. Kammer - Denitrifikation, (4) 2. Kammer - Nitrifikation, (5) Belüftungsanlage, (6) 3. Kammer - Absetzbecken, (7) Ablauf 
Funktionsweise der Kleinkläranlage MINICLAR:

- Die Mikroorganismen bilden im Wirbelbettreaktor Belebtschlammflocken aus.

- Sie reinigen das Wasser ohne Zusatz von Nährstoffen oder Chemikalien.

- In der 1. Kammer findet der anaerobe Abbau von Nitraten und Nitriten statt (Denitrifikation).

- In der 2. Kammer findet der aerobe Abbau von ammoniakalischen Verbindungen statt (Nitrifikation).

- In der 3. Kammer setzt sich der Belebtschlamm ab und wird in die 1. Kammer zurückgepumpt (Nachklärung).

\section{Zusammenfassung}

Für das Bundesland Brandenburg kann zum Ende des Jahres 2002 festgestellt werden, dass in den dichter besiedelten Gebieten und kreisfreien Städten ein guter Anschlussgrad bei der Abwasserversorgung erzielt wurde. Die Reinigungsleistung der vorhanden Abwasseranlagen hat sich deutlich verbessert. Es ist wünschenswert, dass aus Kostengruinden und auch aus umweltpolitischer Sicht in Streusiedlungen und ländlichen Gebieten ein hoher Anschlussgrad an zentrale Anlagen nicht realisiert wird. Deshalb muss die Verwendung dezentraler Lösungen bei der Abwasserentsorgung weiterhin gefördert werden.

\section{Ausblick}

Zum Schluss wollen wir noch kurz auf die vor uns liegenden Aufgaben verweisen. Die Umsetzung der Wasserrahmenrichtlinie 2000/60/EG muss demnächst erfolgen. Die Umsetzung dieser sogenannten Abwasserrichtlinie ist notwendig, um den „guten Zustand“ der brandenburgischen Gewässer teilweise wieder zu erreichen und für die Zukunft zu erhalten. Die Einhaltung der von der EG geforderten Fristen bis 2005 in Brandenburg wird von der Kreditanstalt fuir Wiederaufbau als wichtigster Finanzgeber als schwierig angesehen [5]. Insbesondere im ländlichen Raum sind verstärkt dezentrale Lösungen als alternative Lösungen in der Abwasserentsorgung einzusetzen.

\section{Literatur}

[1] EG-Richtlinie 91/271/EWG vom 25.9.1991 über die Behandlung von kommunalem Abwasser (Kommunalabwasser-Richtlinie), geändert durch die Richtlinie 98/15/EG der Kommission vom 27. Februar 1998

[2] Brandenburgische Kommunalabwasserverordnung (BbgKAbwV) vom 18. Februar 1998 (GVBI II 1998 S. 182)

[3] Ministerium für Landwirtschaft, Umweltschutz und Raumordnung des Landes Brandenburg, Referat P/ÖA, Dr. JensUwe Schade, 2000-2002

[4] PROTEKUM Umweltinstitut GmbH, Lehnitzstr. 73, 16515 Oranienburg

[5] Wolff, G.; Abwasserentsorgung in Deutschland in KfW Beiträge zur Mittelstands- und Strukturpolitik, Brönners Druckerei, Frankfurt a. M., 2000

\section{Autoren}

Prof. Dr. rer. nat. Lothar Ebner

Technische Fachhochschule Wildau

Fachbereich Ingenieurwesen/Wirtschaftsingenieurwesen

Telefon +493375 508-226

E-Mail: protekum@t-online.de

Dr. rer. nat. Michael König

PROTEKUM Umweltinstitut GmbH

Lehnitzstraße 73, 16515 Oranienburg

Telefon +493301698100

E-Mail: protekum@t-online.de

cand. ing. Andrea Wardacki

Fachhochschule Nordhausen

Fachbereich Flächen- und Stoffrecycling

99734 Nordhausen

Dipl.-Ing. Sandra Giern

PROTEKUM Umweltinstitut GmbH

Lehnitzstraße 73, 16515 Oranienburg

Telefon +493301698100

E-Mail: protekum@t-online.de 